\title{
To treat or not to treat drug-refractory epilepsy by the ketogenic diet? That is the question
}

\author{
Marzena Ułamek-Kozioł'1,2, Ryszard Pluta², Anna Bogucka-Kocka ${ }^{3}$, Stanisław J. Czuczwar ${ }^{4,5}$ \\ ${ }^{1}$ First Department of Neurology, Institute of Psychiatry and Neurology, Warsaw, Poland \\ ${ }^{2}$ Laboratory of Ischemic and Neurodegenerative Brain Research, Mossakowski Medical Research Centre, Polish Academy \\ of Sciences, Warsaw, Poland \\ ${ }^{3}$ Department of Biology and Genetics, Medical University of Lublin, Lublin, Poland \\ ${ }^{4}$ Department of Physiopathology, Institute of Rural Health, Lublin, Poland \\ ${ }^{5}$ Department of Pathophysiology, Medical University of Lublin, Lublin, Poland
}

Ułamek-Kozioł M, Pluta R, Bogucka-Kocka A, Czuczwar S J. To treat or not to treat drug-refractory epilepsy by the ketogenic diet? That is the question. Ann Agric Environ Med. 2016; 23(4): 533-536. doi: 10.5604/12321966.1226841

\begin{abstract}
Epilepsy is a serious neurologic disorder worldwide which affects about 1\% of the population (ca. 50 million people), the highest prevalence occurring in both children and elderly. Apart from idiopathic forms, etiology of the disease involves multiple brain risk factors - the most frequent being cerebrovascular diseases, tumours and traumatic injuries. Several treatment options exist, including, for instance, pharmacotherapy, vagal nerve stimulation or epilepsy surgery. In spite of treatment, about $30 \%$ of patients with epilepsy still have seizures and become drug-refractory. This is why other treatment options may be recommended, and ketogenic diet seems a last-chance method, especially in children and adolescents with epilepsy. The diet contains high amounts of fat and low carbohydrates with vitamin supplementation. The elevated concentrations of ketones induced by the diet may result in inhibition of the synaptic activity of glutamate, the mammalian target of the rapamycin pathway, and activation of adenosine triphosphate-sensitive potassium channels. One of the main ketones is acetone, shown to increase the seizure threshold and potentiate the anticonvulsant activity of some antiepileptic drugs. The clinical effectiveness of the ketogenic diet has been confirmed in a number of clinical trials carried out mainly on children. A wider use of the ketogenic diet may be limited by the number of early adverse effects (gastrointestinal distress, acidosis, hypoglycaemia, dehydration and lethargy), and late adverse effects (hyperuricaemia, hyperlipidaemia, kidney stones, easy bruising, and decreases in height and weight). Recently, data are available on the negative impact of the ketogenic diet on the qualitative characteristics of lipoprotein subfractions which points to the atherogenic fenotype as a new side-effect. In conclusion, future research directed to the proper identification of patients (in terms of age, epilepsy type and duration, recommended antiepileptic drugs) is necessary to answer the title question.
\end{abstract}

Key words

ketogenic diet, ketones, acetone, epilepsy, antiepileptic drugs

\section{INTRODUCTION}

Epilepsy is a serious and expensive health problem worldwide [1] and accounts for estimated direct and indirect costs annually of about $€ 15.5$ billion in Europe [2] and \$15.5 billion in the United States [3]. In addition, epilepsy is the most frequent and devastating neurologic disorder in the world, with an estimated prevalence of $1 \%$ of the population [4, 5], affecting 50 million people, $80 \%$ of whom live in poor countries [6]. The highest prevalence occurs in both children and elderly, with low frequency in early adulthood [7]. Different kinds of brain lesions and injuries are involved in an increased risk for the generation of epilepsy. Epileptogenesis is a cascade of events, following genetic mutations, brain infections, traumatic brain injury or status epilepticus, which eventually lead to a conversion of normal into an epileptic brain [8]. Brain alterations are frequently followed by a latent phase of variable length before the appearance

Address for correspondence: Stanisław J.Czuczwar, Department of Physiopathology, Institute of Rural Health, Lublin, Poland

E-mail: czuczwarsj@yahoo.com

Ryszard Pluta,Laboratory of Ischemic and Neurodegenerative Brain Research, Mossakowski Medical Research Centre, Polish Academy of Sciences, Warsaw, Poland E-mail: pluta@imdik.pan.pl

Received: 07 September 2016; accepted: 09 November 2016 of clinical symptoms. The latent stage is characterized by different neurochemical and neuropathological alterations in the brain, such as neuronal necrosis, apoptosis, axonal sprouting, synaptogenesis and induction of gene expression $[9,10]$. Early symptomatic seizures develop at the time of the brain damage and are the marker of a severe injury. Epilepsy is a widespread and life-threatening neurological disease characterized by spontaneous sudden, irregular and abnormal neuronal discharges. Epilepsy is characterized by seizures that reflect a repeated neuropathological and uncontrolled electric activity in one or a few regions of the brain [11]. The etiology of epilepsy is complex and associated with multiple brain risk factors, the most common including cerebrovascular diseases (21\%), tumours (11\%), traumatic brain injuries (7\%), and others like toxic and infectious disorders, congenital malformations, and genetic alternations $[9,10]$. The development of epileptic neuropathology causes cognitive, psychological and social consequences [11]. The neuropathological mechanisms of epilepsy are only partly understood and may include a general neurochemical and blood-brain barrier dysfunction, generation of free radicals and the release of excitotoxins, such as glutamate, and alternations in energy metabolism [9]. Such a neuropathology is believed to be the end result of long-term alterations in the brain neuronal network [9]. 
Classic epilepsy therapy includes pharmacologic treatment, vagal nerve stimulation and epilepsy surgery. Despite these therapies, around $30 \%$ of patients with epilepsy are not sufficiently controlled and become drug-refractory [7]. This is defined as an insufficient control of seizures despite an optimal therapy with a combination of 2 or even more properly selected antiepileptic drugs [12]. Patients with drugrefractory epilepsy depend on informal family and friends' care, and on healthcare professionals, such as neurologists, psychologists, social workers, etc. Complications due to drug-refractory epilepsy in children and adolescents result in repeated hospitalizations and the institutionalization of many patients. Living with uncontrolled epilepsy has a harmful impact on the quality of the lives of the children and their caregivers.

Ketogenic diet. Ketogenic diet therapy should be considered in patients who have insufficiently responded to the therapy of two well-selected and well-dosed antiepileptic drugs [13]. In these circumstances, adding extra antiepileptic drugs frequently does not result in considerable seizure reduction. This is why other therapies, such as diet, including the ketogenic diet, are often recommended by medical neurologists to place the patients under better control [9, 14]. The ketogenic diet is a last-chance treatment for many children and adolescents with epilepsy. Although epilepsy study is flourishing, the mechanisms of this disorder have not yet been completely solved, and fully effective treatments in all patients with epilepsy have not been discovered todate. Epilepsy is a top priority for many pharmaceutical companies, making epilepsy one of the most investigated brain disorders in the pharmaceutical industry. It should be realized that in spite of the annual development of new antiepileptic drugs, the treatment, as already mentioned, in about one-third of epileptic subjects is not fully effective $[15,16]$. For numerous refractory epilepsy patients, a dietary treatment offers hope for an improved quality of lives with a considerable decrease in seizure frequency. For this reason, an increase of the worldwide use of the ketogenic diet is observed at the moment [17]. A successful realization of this dietary therapy depends on active support from a healthcare team, social and educational system, and finally the family [17]. The ketogenic diet requires a strict dietetic and medical control because of its restrictiveness and side-effects (Tab. 1) [13].

Ketogenic diet - mechanisms of action. As its principal working mechanism has not yet been fully understood, it is not clear which patients benefit most [18]. One of the probable working mechanisms of the ketogenic diet is inhibition of glutamatergic excitatory transmission in synapses [19]. Ketones block glutamate transport into synaptic vesicles. Other mechanisms include carbohydrate reduction, activation of adenosine triphosphate-sensitive potassium channels by mitochondrial metabolism, and inhibition of the mammalian target of the rapamycin pathway [20]. It is certain that one of the main ketone bodies derived from the diet is acetone, exerting a clear-cut anticonvulsant activity against pentylenetetrazol- or 4-aminopyridine-induced seizures in mice, and at the effective anticonvulsant doses, the ketone produced no motor impairment [21]. Probably, the protective activity of acetone is not associated with its metabolites, acetol, 1,2-propanediol or pyruvic acid
Table 1. Side-effects in children with drug-refractory epilepsy treated by ketogenic diet $[4,7,29,32-35,40]$.Modified and updated from Lee and Kossoff [41]

\begin{tabular}{|c|c|c|}
\hline Early side-effects & Late side-effects & Rare side-effects \\
\hline Acidosis & $\begin{array}{l}\text { Alternations in } \\
\text { apolipoprotein B }\end{array}$ & Allergy \\
\hline Constipation & $\begin{array}{l}\text { Alternations in arterial } \\
\text { function }\end{array}$ & $\begin{array}{l}\text { Basal ganglia } \\
\text { changes }\end{array}$ \\
\hline Dehydration & Atherogenic phenotype & Cardiomyopathy \\
\hline $\begin{array}{l}\text { Exacerbation of } \\
\text { gastrooesophageal reflux } \\
\text { disease }\end{array}$ & Bone fractures & Oesophagitis \\
\hline Excessive ketosis & Bruising & $\begin{array}{l}\text { Fanconi's renal } \\
\text { tubular acidosis }\end{array}$ \\
\hline Fatigue & $\begin{array}{l}\text { Decreased bone mineral } \\
\text { density }\end{array}$ & Gallstones \\
\hline Food refusal & Dyslipidaemia & Hepatitis \\
\hline Gastrointestinal distress & Hyperuricaemia & Hypocalcaemia \\
\hline Hypoglycaemia & Kidney stones & Hypomagnesaemia \\
\hline Increased seizure frequency & Poor growth & Hyponatraemia \\
\hline Lethargy & $\begin{array}{l}\text { Secondary carnitine } \\
\text { deficiency }\end{array}$ & Hypoproteinaemia \\
\hline Vomiting & $\begin{array}{l}\text { Vitamin D deficiency } \\
\text { Vitamin, mineral and enzyme } \\
\text { deficiencies, } \\
\text { Weight loss }\end{array}$ & $\begin{array}{l}\text { Increased illnesses } \\
\text { Selenium deficiency } \\
\text { Lipoid pneumonia } \\
\text { Sudden death }\end{array}$ \\
\hline
\end{tabular}

[21]. Interestingly, this ketone body could also affect the anticonvulsant activity of a number of antiepileptic drugs against maximal electroshock-induced seizures in mice. When administered in subprotective doses against maximal electroshock seizure threshold tests, it efficiently potentiated the protective activity of carbamazepine, phenobarbital, valproate (classic antiepileptic drugs) and lamotrigine (a newer antiepileptic drug) against maximal electroshockinduced convulsions in mice with the use of supramaximal current intensity. Pharmacokinetic interactions between acetone and antiepileptics seem unlikely because the ketone did not modify the total brain concentrations of the affected antiepileptic drugs. However, the anticonvulsant activity of some antiepileptic drugs was not influenced by acetone - these are phenytoin (a classic antiepileptic) and both, oxcarbazepine and topiramate (newer antiepileptic drugs) [22]. Apart from acetone, other constituents of the ketogenic diet may also be taken into consideration in terms of the anticonvulsant activity. For instance, caprylic acid (an 8 carbon fatty acid, present in the medium-chain triglyceride ketogenic diet) elevated the threshold for $6 \mathrm{~Hz}$ psychomotor seizures in rodents, but was ineffective as regards the maximal electroshock threshold seizure test. It is noteworthy that caprylic acid, although ineffective against electroconvulsions, significantly enhanced the protection offered by valproate against maximal electroshock-induced seizures in mice, without any involvement of a pharmacokinetic mechanism. This positive interaction was also noted against $6 \mathrm{~Hz}$-induced convulsions in mice [23]. Another constituent of the medium chain triglyceride ketogenic diet, capric acid, a 10-carbon fatty acid, has also been found to be effective against a number of convulsive tests in mice - maximal electroshock seizure threshold, $6 \mathrm{~Hz}$ seizure threshold and intravenous pentylenetetrazol seizure tests [24]. Recently, an intriguing hypothesis has appeared that caprylic and capric acid elevated 
the brain concentration of tryptophane, as well as the central and peripheral level of kynurenic acid. Interestingly, when the passage of tryptophane into the brain was prevented, the anticonvulsant activity of these fatty acids disappeared [25].

Clinical effectiveness and adverse effects. The ketogenic diet is high in fat and low in carbohydrates, and has an adequate amount of protein for growth, usually at a 4:1 fat to protein ratio, carbohydrates plus required vitamin supplementation $[4,5]$. The ketogenic diet has been proved to be useful in children with epilepsy by a meta-analysis study and in randomized controlled trials: $15 \%$ of patients became seizure-free and 33\% reached a 50\% decrease in seizure frequency [26-29]. If a reduction in seizure frequency is reached, antiepileptic drug therapy decrease can be taken into account after 3-6 months. Patients usually stay on the diet for 1-2 years before discontinuation is considered [30]. A recent study of the ketogenic diet in adults with refractory epilepsy has additionally demonstrated a high efficacy and tolerability [31]. Firstly, there was a lot of enthusiasm because patients tolerated the therapy very well, without dangerous side-effects. Fast enthusiasm went down because of early noted side-effects which included gastrointestinal distress, acidosis, hypoglycaemia, dehydration and lethargy (Tab. 1) [7, 29, 32-34]. Late side-effects, including hyperuricaemia, hyperlipidaemia, kidney stones, easy bruising, and decreases in height and weight were aditionally observed (Tab. 1) [7, 32-34]. Also, decreased bone density and bone fractures were frequently noted [7, 32-34]. Deaths were recorded in children during the ketogenic diet therapy, while it is not clear whether any of the noted deaths were directly associated with using the ketogenic diet [7, 32, 33]. Side-effects of the ketogenic diet from time-to-time necessitated stopping the treatment because of anaphylaxis (Tab. 1) [4], and it is very important for pediatric neurologists to recognize this. Nowadays, use of the ketogenic diet treatment for children and adolescents with intractable epilepsy continues to grow in attractiveness, both from clinical and research point of view. Recently, de Lima et al. [35] suggested that the diet had an atherogenic end result on the physical properties of lipoproteins subfractions, regardless of the positive effect of the ketogenic diet therapy on refractory seizure control in children and adolescents. Their data confirmed the positive clinical effect of the ketogenic diet therapy on refractory epilepsy, the effect of which is unquestionable. However, they stressed the negative impact of the diet on the qualitative characteristics of lipoprotein subtractions. Following use of the ketogenic diet, the percentage of small LDL increased, and both the LDL size and percentage of small HDL decreased. The ketogenic diet caused development of atherogenic phenotype in patients: 3 and 6 months following the treatment, and was evident in $31 \%$ and $52 \%$ of cases, respectively. In another study, the initial alterations in lipids, apolipoprotein B and arterial function observed within the first year of the ketogenic diet therapy were found to be reversible, and not significant after 2 years of therapy [36]. The dyslipidaemia observed after ketogenic diet therapy is associated not only with the ketogenic diet, but also with the drugs used during the treatment of epilepsy [37]. The atherogenic side-effect observed by de Lima et al. [35] seriously challenges the use of the ketogenic diet therapy for refractory epilepsy. In 2015, de Lima et al. [38] also predicted that such a therapy probably contributed to changes in the size of lipoproteins, particularly related to small LDL. In their recent study, they highlighted for the first time the importance of monitoring not only the classic lipid profile, but also the size of lipoprotein subfractions [35]. An important change in the mindset of neurologists and dietitians at ketogenic diet centres is now to prevent these negative side effects before they actually occur. Finally the authors suggested some practical observations for clinicians [35]: First, use a modified ketogenic diet, with fewer atherogenic fatty acids. Second, initiate coadjuvant ketogenic diet treatment early to reduce the number and quantity of antiepileptic drugs that show dyslipidemic action. Finally, include lipoprotein size in routine laboratory examinations in addition to the classic lipid profile [35].

\section{CONCLUSIONS}

The study by de Lima et al. [35], however, has some limitations. The number of individuals investigated was relatively small, although the number of measurements made was quite large and the differences clearly present. Future investigations also need to determine ways on how to improve the quality of the lives of patients in the later course of the illness. Effective integration is needed of these new side-effects with the known heterogeneity of refractory epilepsy. It is probable that this study should have had a control group with healthy patients versus the ketogenic diet, and the patients without antiepileptic drugs but also with the ketogenic diet [39]. The investigated group should be more homogeneous in age. The researchers should also investigate the vascular function using, e.g. carotid ultrasound in the future. However, the Lima's et al. [35] study sheds new light on the complex relationship between the ketogenic diet therapy and refractory epilepsy; it also generates a number of questions that need to be answered. Is this treatment a really safe therapy? What facts continue to speak in support of the ketogenic diet treatment? Obviously, regardless of the early enthusiasm for the ketogenic diet therapy, we are still in the early stages of safe use and understanding it. We should draw conclusions from the study by de Lima et al. [35] and return to the bench to do our homework before negative side-effects can be noted. This study should also serve as a fresh starting point for clinicians interested in developing the ketogenic diet as an alternative therapy for the refractory epilepsy. This approach should be perceived as a new ground for the design and implementation of clinical research to help answer some of the numerous questions remaining. We can add an atherogenic reaction to the dangerous risk sideeffects of the ketogenic diet treatment. For this goal, larger multicentre studies are needed. Future research should focus on identifying patients (according to age, type and duration of epilepsy, drug therapy, etc.) who benefit the most from the ketogenic diet. Can refractory epilepsy be treated by the ketogenic diet? Currently, this question has no answer; we can only add the atherogenic phenotype to new side-effects.

\section{Acknowledgements}

The authors acknowledge the support provided by the Mossakowski Medical Research Centre, Polish Academy of Sciences, Poland (T3-RP), and by the statutory grants from the Institute of Rural Health and the Medical University in Lublin, Poland (SJC). 


\section{REFERENCES}

1. Strzelczyk A, Reese J P, Dodel R, Hamer H M. Cost of epilepsy: a systematic review. Pharmacoeconomics 2008; 26(6): 463-476.

2. de Kinderen R J A, Lambrechts D A J, Wijnen B F M, Postulart D, Aldenkamp A P, Majoie M H, et al. An economic evaluation of the ketogenic diet versus care as usual in children and adolescents with intractable epilepsy: An interim analysis. Epilepsia 2016; 57(1): 41-50.

3. Cramer J A, Wang Z J, Chang E, Copher R, Cherepanov D, Broder M $\mathrm{S}$. Health-care costs and utilization related to long- or short-acting antiepileptic monotherapy use. Epilepsy Behav. 2015; 44: 40-46.

4. Levy Y, Peleg-Weiss L, Goldberg-Stern H. The modified Atkins diet for intractable epilepsy may be associated with late-onset egg-induced anaphylactic reaction: a case report. Nutrition. 2011; 27(3): 380-382.

5. Pluta R, Jabłoński M. The ketogenic diet for epilepsy therapy in children: quo vadis? Nutrition 2011;27(5):615-616.

6. www.who.int/mediacentre/factsheets/fs999/en/

7. Zupec-Kania BA, Spellman E. An overview of the ketogenic diet for pediatric epilepsy. Nutr Clin Pract 2008;23(6):589-96.

8. Radzik I, Miziak B, Dudka J, Chrościńska-Krawczyk M, Czuczwar S J. Prospects of epileptogenesis prevention. Pharmacol Rep. 2015 67(3): 663-668.

9. Herman S T. Epilepsy after brain insult. Targeting epileptogenesis. Neurology 2002; 59(Suppl 5): S21-S26.

10. Gitaí D L, Romcy-Pereira R N, Gitaí L L, Leite J P, Garcia-Cairasco N, Paço-Larson M L. Genes and epilepsy I: epilepsy and genetic alterations. Rev Assoc Med Bras. 2008; 54(3): 272-278.

11. Fisher R S, Acevedo C, Arzimanoglou A, Bogacz A, Cross J H, Elger C E, et al. ILAE official report: a practical clinical definition of epilepsy. Epilepsia 2014; 55(4): 475-82.

12. Kwan P, Arzimanoglou A, Berg A T, Brodie M J, Allen Hauser W, Mathern G, et al. Definition of drug resistant epilepsy: consensus proposal by the ad hoc Task Force of the ILAE Commission on Therapeutic Strategies. Epilepsia 2010; 51(6): 1069-1077.

13. Kossoff E H, Zupec-Kania B A, Amark P E, Ballaban-Gil K R, Christina Bergqvist A G, Blackford R, et al. Charlie Foundation, Practice Committee of the Child Neurology Society; Practice Committee of the Child Neurology Society; International Ketogenic Diet Study Group. Optimal clinical management of children receiving the ketogenic diet: recommendations of the International Ketogenic Diet Study Group. Epilepsia 2009; 50(2): 304-317.

14. Brodie M J, Barry S J, Bamagous G A, Norrie J D, Kwan P. Patterns of treatment response in newly diagnosed epilepsy. Neurology 2012; 78(20): 1548-1554.

15. Sillanpää M, Schmidt D. Natural history of treated childhood-onset epilepsy: prospective, long-term population-based study. Brain 2006; 129(3): 617-624

16. Czuczwar S J, Patsalos P N. The new generation of GABA enhancers. Potential in the treatment of epilepsy. CNS Drugs 2001; 15(5): 339-350.

17. Kossoff E H, McGrogan J R. Worldwide use of the ketogenic diet. Epilepsia 2005; 46(2): 280-289.

18. Casey J C, McGrogan J, Pillas D, Pyzik P, Freeman J, Vining E P. The implementation and maintenance of the ketogenic diet in children. J Neurosci Nurs. 1999; 31(5): 294-302.

19. Vehmeijer F O, van der Louw E J, Arts W F, Catsman-Berrevoets C E Neuteboom R F. Can we predict efficacy of the ketogenic diet in children with refractory epilepsy? Eur J Paediatr Neurol. 2015; 19(6): 701-715.

20. Danial N N, Hartman A L, Stafstrom C E, Thio L L. How does the ketogenic diet work? Four potential mechanisms. J Child Neurol. 2013; 28(8): 1027-1033.

21. Gasior M, French A, Joy M T, Tang R S, Hartman A L, Rogawski M A. The anticonvulsant activity of acetone, the major ketone body in the_ketogenic diet, is not dependent on its metabolites acetol, 1,2-propanediol, methylglyoxal, or pyruvic acid. Epilepsia 2007; 48(4): $793-800$

22. Zarnowska I, Luszczki J J, Zarnowski T, Buszewicz G, Madro $\mathrm{R}$, Czuczwar S J, et al. Pharmacodynamic and pharmacokinetic interactions between common antiepileptic drugs and acetone, the chief anticonvulsant ketone body elevated in the ketogenic diet in mice. Epilepsia 2009; 50(5): 1132-1140.

23. Wlaź P, Socała K, Nieoczym D, Łuszczki J J, Zarnowska I, Zarnowski $\mathrm{T}$, et al. Anticonvulsant profile of caprylic acid, a main constituent of the medium-chain triglyceride (MCT) ketogenic diet, in mice. Neuropharmacology 2012; 62(4): 1882-1889.

24. Wlaź P, Socała K, Nieoczym D, Żarnowski T, Żarnowska I, Czuczwar $S \mathrm{~J}$, et al. Acute anticonvulsant effects of capric acid in seizure tests in mice. Prog Neuropsychopharmacol Biol Psychiatry 2015; 57: 110-116.

25. Maciejak P, Szyndler J, Turzyńska D, Sobolewska A, Kołosowska K, Krząścik P, et al. Is the interaction between fatty acids and tryptophan responsible for the efficacy of a ketogenic diet in epilepsy? The new hypothesis of action. Neuroscience 2016; 313: 130-48.

26. Henderson C B, Filloux F M, Alder S C, Lyon J L, Caplin D A. Efficacy of the ketogenic diet as a treatment option for epilepsy: meta-analysis. J Child Neurol. 2006; 21(3): 193-198.

27. Keene D L. A systematic review of the use of the ketogenic diet in childhood epilepsy. Pediatr Neurol. 2006; 35(1): 1-5.

28. Neal E G, Chaffe H, Schwartz R H, Lawson M S, Edwards N, Fitzsimmons $\mathrm{G}$, et al. The ketogenic diet for the treatment of childhood epilepsy: a randomized controlled trial. Lancet Neurol. 2008; 7: 500-506.

29. Lambrechts D A J E, de Kinderen R J A, Vles J S H, de Louw A J A, Aldenkamp A P, Majoie H J M. A randomized controlled trial of the ketogenic diet in refractory childhood epilepsy Acta Neurol Scand. 2016 DOI: 10.1111/ane.12592.

30. Hsieh D T, Pfeifer H H, Thiele E A. Dietary management of epilepsy. Am Fam Physician. 2012; 86: 1000-1001.

31. Klein P, Janousek J, Barber A, Weissberger R. Ketogenic diet treatment in adults with refractory epilepsy. Epilepsy Behav. 2010; 19: 575-579.

32. Freeman J M, KossoffE H, Hartman A L. The ketogenic diet: one decade later. Pediatrics 2007; 119: 535-543.

33. Hartman A L, Vining E P G. Clinical aspects of the ketogenic diet. Epilepsia 2007; 48: 31-42.

34. Kossoff E H, Hartman A L. Ketogenic diets: New advances for metabolism-based therapies. Curr Opin Neurol. 2012; 25: 173-178.

35. De Lima P A, Prudencio M B, Murakami D K, de Brito Sampaio L P, Neto A M F, Damasceno N R T. Effect of classic ketogenic diet treatment on lipoprotein subfractions in children and adolescents with refractory epilepsy. Nutrition 2017; 33: 271-277.

36. Kapetanakis M, Liuba P, Odermarsky M, Lundgren J, Hallbook T. Effects of ketogenic diet on vascular function. Europ J Paediatr Neurol. 2014; 18: 489-494.

37. Vyas M V, Davidson B A, Escalaya L, Costella J, Saposnik G, Burneo J G. Antiepileptic drug use for treatment of epilepsy and dyslipidemia: Systematic review. Epilepsy Res. 2015; 113: 44-67.

38. Lima PA, de Brito Sampaio L P, Damasceno N R. Ketogenic diet in epileptic children: impact on lipoproteins and oxidative stress. Nutr Neurosci. 2015; 18: 337-344.

39. Wang H S, Lin K L. Ketogenic diet: An early option for epilepsy treatment, instead of a last choice only. Biomed J. 2013; 36: 16-17.

40. Martin K, Jackson CF, Levy RG, Cooper PN. Ketogenic diet and other dietary treatments for epilepsy. Cochrane Database Syst Rev. 2016 Feb 9; 2: CD001903.

41. Lee P R, Kossoff E H. Dietary treatments for epilepsy: Management guidelines for the general practitioner. Epilepsy Behav. 2011; 21: 115-121. 